PROCEEDINGS OF THE

AMERICAN MATHEMATICAL SOCIETY

Volume 132, Number 2, Pages 463-471

S 0002-9939(03)07259-9

Article electronically published on August 28, 2003

\title{
ON A CLASS OF ELLIPTIC FUNCTIONS ASSOCIATED WITH IMAGINARY QUADRATIC FIELDS
}

\author{
LI-CHIEN SHEN
}

(Communicated by David E. Rohrlich)

\begin{abstract}
Let $-D$ be the field discriminant of an imaginary quadratic field. We construct a class of elliptic functions associated naturally with the quadratic field $Q(\sqrt{-D})$ which, combined with the general theory of elliptic functions, allows us to provide a unified theory for two fundamental results (one classical and one due to Ramanujan) about the elliptic functions.
\end{abstract}

\section{$\S 1$. INTRODUCTION}

Throughout this paper, the following notation will be adopted. The letter $D$ will always denote a positive integer with the property that $-D$ is a discriminant of a quadratic field and $D^{\times}$denotes the reduced residue system modulo $D$. There are three summation symbols: $\sum^{\times}$denotes the sum over the reduced residue system $D^{\times}, \sum$ denotes the sum over the set of positive integers and $\sum^{\prime}$ denotes the sum over the set of integers with the zero ${ }^{\text {th }}$ term excluded. Throughout the paper, the differentiation is always with respect to the variable $z$. We will always assume that $\tau$ is a complex number such that $\operatorname{Im} \tau>0$ and $q=e^{i \pi \tau}$. The symbol $\theta_{1}(z \mid \tau)$ denotes the theta function

$$
\theta_{1}(z \mid \tau)=2 q^{\frac{1}{4}} \sum(-1)^{n+1} q^{n(n-1)} \sin (2 n-1) z .
$$

The notation $\frac{\theta_{1}^{\prime}}{\theta_{1}}(z \mid \tau)$ is an abbreviation for $\frac{\theta_{1}^{\prime}(z \mid \tau)}{\theta_{1}(z \mid \tau)}$.

Let $-D$ be the discriminant of an imaginary quadratic field. Let $\chi$ be a primitive character modulo $D$. Define

$$
Q_{\chi, D}(t)=\sum^{\times} \chi(k) \frac{t^{k}}{1-t^{D}}
$$

and if $\chi(k)=(-D \mid k)$, the Kronecker symbol, we simply denote it by $Q_{D}(t)$. We will consider the function

$$
\varphi\left(z \mid \frac{D \tau}{2}\right)=h z+\frac{w}{2} \sum \frac{1}{n} Q_{D}\left(q^{n}\right) \sin 2 n z,
$$

where $h$ and $w$ denote, respectively, the class number and the number of units in the quadratic field $Q(\sqrt{-D})$. We recall that $w=2$ if $D \geq 7 ; w=4$ for $D=4 ; w=6$ for $D=3$.

Received by the editors October 3, 2002.

2000 Mathematics Subject Classification. Primary 33E05.

Key words and phrases. Elliptic function, character, class number, quadratic field, discriminant. 
The consideration of $\varphi\left(z \mid \frac{D \tau}{2}\right)$ is motivated by the following two examples:

$$
\begin{gathered}
\varphi\left(z \mid \frac{3 \tau}{2}\right)=z+3 \sum \frac{1}{n} \frac{q^{n}}{1+q^{n}+q^{2 n}} \sin 2 n z \\
\varphi\left(z \mid \frac{4 \tau}{2}\right)=z+2 \sum \frac{1}{n} \frac{q^{n}}{1+q^{2 n}} \sin 2 n z
\end{gathered}
$$

We remark that $\varphi\left(z \mid \frac{4 \tau}{2}\right)$ is well known and classical and $\varphi\left(z \mid \frac{3 \tau}{2}\right)$ is due to Ramanujan (see [3] and [4]). The main result of this work is the following theorem, and its implication will be discussed in the last section of the paper.

Theorem 1. Let $-D$ be the discriminant of a quadratic field. Then

$$
\exp \left(\frac{-4 i \varphi(z \mid \tau)}{w}\right)=(-1)^{\frac{\phi(D)}{2}} e^{i z\left(\phi(D)-\frac{4 h}{w}\right)} \prod_{k \in R} \frac{\theta_{1}\left(z+\frac{k \pi \tau}{D} \mid \tau\right)}{\theta_{1}\left(z-\frac{k \pi \tau}{D} \mid \tau\right)},
$$

where $\phi(D)$ is the Euler phi-function which counts the number of elements in $D^{\times}$ and $R=\left\{k \in D^{\times}:(-D \mid k)=1\right\}$.

Moreover, $\exp (-2 i \varphi(z \mid \tau))$ is elliptic with periods $\pi$ and $\pi \tau$.

For later use we also define $N=\left\{n \in D^{\times}:(-D \mid n)=-1\right\}$. We note that if $D=p$, a prime, then $R$ is the set of all the quadratic resides modulo $p$ and $N$ the set of quadratic non-residues modulo $p$.

We remark that the function $Q_{\chi, D}(t)$ arises naturally from the Dirichlet $L$ series via the Mellin transform. To see this, we write

$$
\sum \chi(n) q^{n}=\sum^{\times} \sum \chi(k D+n) q^{k D+n}=\frac{\sum^{\times} \chi(n) q^{n}}{1-q^{D}} .
$$

With $q=e^{-t}$, the Dirichlet $L$ series $L(s, \chi)=\sum \frac{\chi(n)}{n^{s}}$ is precisely the Mellin transform of the left-hand side of (1.2) (see [4] for details).

The general properties of the primitive characters can be found in [1, pp. 343349]; for this work we only need the following facts:

(A) $\chi(k)=0$ if $(k, D)>1$ and $\chi(k+D)=\chi(k)$.

(B) $\sum^{\times} \chi(k)=0$.

(C) $h=-\frac{w}{2} \sum^{\times}(-D \mid k) \frac{k}{D}$.

(D) Let $\varsigma=e^{\frac{2 i \pi}{D}}$. Define the Gauss sum $g_{k}(\chi)=\sum^{\times} \chi(n) \varsigma^{n k}$ and let $g(\chi)=$ $g_{1}(\chi)$. Then $g_{k}(\chi)=\bar{\chi}(k) g(\chi)$ if $(k, D)=1$ and $g_{k}(\chi)=0$ if $(k, D)>1$, where $\bar{\chi}$ denotes the complex conjugate of $\chi$.

The content of this work is organized as follows. Theorem 1 is proved in Section 2. In Section 3, we derive an identity relating $\sigma_{1}(n)$ (the sum of the divisors of $n$ ) with the evaluation of the Weierstrass elliptic function at the rational points of its period parallelogram. In Section 4, we introduce the notion of an elliptic function associated with the imaginary quadratic fields and discuss the relevance of the two above-mentioned classical and Ramanujan functions within this framework.

The reader's familiarity with the basic properties of the theta functions is assumed (see Chapter 21 of [6] ). 


\section{$\S 2$. Proof of Theorem 1}

We begin with a lemma connecting the logarithmic derivative $\theta_{1}^{\prime} / \theta_{1}$ with the derivative of the function $\varphi$ defined in (1.1). For the purpose of later use, we will prove a more general result involving the character $\chi$.

Lemma 2.1. If $\chi(-1)=-1$, then

$$
\sum^{\times} \chi(k) \frac{\theta_{1}^{\prime}}{\theta_{1}}\left(z+\frac{k \pi \tau}{2} \mid \frac{D \tau}{2}\right)=4 \sum Q_{\chi, D}\left(q^{n}\right) \cos 2 n z .
$$

Proof. The proof is based on the identity:

$$
i \frac{\theta_{1}^{\prime}}{\theta_{1}}(z \mid \tau)=1+2 \sum^{\prime} \frac{e^{2 i n z}}{1-q^{2 n}},
$$

where the complex number $z$ satisfies the requirement: $|q|<\left|e^{i z}\right|<1$.

Before applying (2.1), we establish some crucial properties first.

Recall that

$$
\theta_{1}(z+\pi \tau \mid \tau)=-q^{-1} e^{-2 i z} \theta_{1}(z \mid \tau)
$$

Then

$$
\frac{\theta_{1}^{\prime}}{\theta_{1}}(z+\pi \tau \mid \tau)=-2 i+\frac{\theta_{1}^{\prime}}{\theta_{1}}(z \mid \tau)
$$

Hence

$$
\frac{\theta_{1}^{\prime}}{\theta_{1}}\left(z+(D-k) \frac{\pi \tau}{2} \mid \frac{D \tau}{2}\right)=-2 i+\frac{\theta_{1}^{\prime}}{\theta_{1}}\left(z-\frac{k \pi \tau}{2} \mid \frac{D \tau}{2}\right) .
$$

We also need the following identity:

$$
\begin{aligned}
\sum^{\times} & \chi(k) \frac{q^{-n k}}{1-q^{-n D}}=-\sum^{\times} \chi(k) \frac{q^{n(D-k)}}{1-q^{n D}} \\
& =\sum^{\times} \chi(D-k) \frac{q^{n(D-k)}}{1-q^{n D}} \quad(\text { since } \chi(-1)=-1) \\
& =\sum^{\times} \chi(k) \frac{q^{n k}}{1-q^{n D}},
\end{aligned}
$$

and the fact that the left-hand side of Lemma 2.1 is even:

$$
\begin{aligned}
\sum^{\times} \chi & (k) \frac{\theta_{1}^{\prime}}{\theta_{1}}\left(-z+\frac{k \pi \tau}{2} \mid \frac{D \tau}{2}\right) \\
& =-\sum^{\times} \chi(k) \frac{\theta_{1}^{\prime}}{\theta_{1}}\left(z-\frac{k \pi \tau}{2} \mid \frac{D \tau}{2}\right) \quad\left(\text { since } \frac{\theta_{1}^{\prime}}{\theta_{1}} \text { is odd }\right) \\
& =\sum^{\times} \chi(-k) \frac{\theta_{1}^{\prime}}{\theta_{1}}\left(z-\frac{k \pi \tau}{2} \mid \frac{D \tau}{2}\right) \quad(\text { since } \chi(-1)=-1) \\
& =\sum^{\times} \chi(D-k) \frac{\theta_{1}^{\prime}}{\theta_{1}}\left(z+(D-k) \frac{\pi \tau}{2} \mid \frac{D \tau}{2}\right) \quad(\text { from }(\mathrm{A}),(\mathrm{B}) \text { and }(2.3)) \\
& =\sum^{\times} \chi(k) \frac{\theta_{1}^{\prime}}{\theta_{1}}\left(z+\frac{k \pi \tau}{2} \mid \frac{D \tau}{2}\right) .
\end{aligned}
$$

If we replace $z$ with $z+\frac{k \pi \tau}{2}$ and $\tau$ with $\frac{D \tau}{2}$, then (2.1) becomes

$$
i \frac{\theta_{1}^{\prime}}{\theta_{1}}\left(z+\frac{k \pi \tau}{2} \mid \frac{D \tau}{2}\right)=1+2 \sum^{\prime} e^{2 i n z} \frac{q^{k n}}{1-q^{D n}} .
$$


Hence

$$
\begin{aligned}
i \sum^{\times} \chi(k) \frac{\theta_{1}^{\prime}}{\theta_{1}}\left(z+\frac{k \pi \tau}{2} \mid \frac{D \tau}{2}\right) & =2 \sum^{\prime} Q_{\chi, D}\left(q^{n}\right) e^{2 i n z} & & (\text { from }(\mathrm{B})) \\
& =2 \sum^{\prime} Q_{\chi, D}\left(q^{n}\right) \cos 2 n z & & (\text { from }(2.5)) \\
& =4 \sum Q_{\chi, D}\left(q^{n}\right) \cos 2 n z & & (\text { from }(2.4)) .
\end{aligned}
$$

This completes the proof of Lemma 2.1.

We now come to the proof of Theorem 1.

Choose $\chi(n)=(-D \mid n)$ in Lemma 2.1. Then

$$
\varphi^{\prime}\left(z \mid \frac{D \tau}{2}\right)=h+w \sum Q_{D}\left(q^{n}\right) \cos 2 n z=h+\frac{w i}{4} \sum^{\times}(-D \mid n) \frac{\theta_{1}^{\prime}}{\theta_{1}}\left(z+\frac{n \pi \tau}{2} \mid \frac{D \tau}{2}\right) .
$$

Integrating (2.7) and replacing $\frac{D \tau}{2}$ by $\tau$, we obtain, after exponentiation,

$$
e^{-4 i \varphi(z \mid \tau) / w}=e^{4 i h z / w} \frac{\prod_{k \in R} \theta_{1}\left(z+\frac{k \pi \tau}{D} \mid \tau\right) / \theta_{1}\left(\frac{k \pi \tau}{D} \mid \tau\right)}{\prod_{n \in N} \theta_{1}\left(z+\frac{n \pi \tau}{D} \mid \tau\right) / \theta_{1}\left(\frac{n \pi \tau}{D} \mid \tau\right)} .
$$

We note that if $\chi(-1)=-1$, then $\chi(D-n)=\chi(-n)=-\chi(n)$. Thus, for $\chi(n)=$ $(-D \mid n)$, as $n$ runs through $N, D-n$ runs through $R$. From this fact and (2.2), it is easy to see that

$$
\prod_{n \in N} \frac{\theta_{1}\left(z+\frac{n \pi \tau}{D} \mid \tau\right)}{\theta_{1}\left(\frac{n \pi \tau}{D} \mid \tau\right)}=(-1)^{\phi(D) / 2} e^{-i \phi(D) z} \prod_{k \in R} \frac{\theta_{1}\left(z-\frac{k \pi \tau}{D} \mid \tau\right)}{\theta_{1}\left(\frac{k \pi \tau}{D} \mid \tau\right)} .
$$

Substituting this into (2.8), we obtain

$$
u(z \mid \tau):=e^{-4 i \varphi(z \mid \tau) / w}=(-1)^{\phi(D) / 2} e^{i z(\phi(D)-4 h / w)} \prod_{k \in R} \frac{\theta_{1}\left(z+\frac{k \pi \tau}{D} \mid \tau\right)}{\theta_{1}\left(z-\frac{k \pi \tau}{D} \mid \tau\right)}
$$

We claim that $\pi \tau$ is a period of $u(z \mid \tau)$. To see this we note that, from (2.2),

$$
u(z+\pi \tau)=u(z) \exp \left\{-4 \sum_{k \in R} \frac{k}{D}+\phi(D)-\frac{4 h}{w}\right\}
$$

and from $(\mathrm{C})$,

$$
\begin{aligned}
-\frac{2 h}{w} & =\sum^{\times}(-D \mid k) \frac{k}{D} \\
& =\sum_{k \in R} \frac{k}{D}-\sum_{n \in N} \frac{n}{D} \\
& =\sum_{k \in R} \frac{k}{D}-\sum_{k \in R} \frac{(D-k)}{D} \\
& =2 \sum_{k \in R} \frac{k}{D}-\frac{\phi(D)}{2} .
\end{aligned}
$$

Hence $u(z+\pi \tau \mid \tau)=u(z \mid \tau)$ and clearly, from (1.1), $\exp \{-2 i \varphi(z \mid \tau)\}=(u(z \mid \tau))^{\frac{w}{2}}$ is of period $\pi$. 
This completes the proof of Theorem 1.

Remark 1. We mention another class of elliptic functions connected to the quadratic fields in a similar fashion. Let $p \geq 7$ be a prime and $p \equiv 3 \bmod 4$. Hence $-p$ is a discriminant of the imaginary quadratic field $Q(\sqrt{-p})$. Based on a fact that if $p$ is prime, the class number of the quadratic field $Q(\sqrt{-p})$ is odd [2, p. 187], we prove that

$$
v(z)=\frac{\prod_{k \in R} \theta_{1}\left(z+\frac{k \pi}{p} \mid \tau\right)}{\left(\theta_{1}(z \mid \tau)\right)^{\frac{p-1}{2}}}
$$

is elliptic and of periods $\tau$ and $\pi \tau$.

We note that since $\theta_{1}(z \mid \tau)$ is of period $\pi, v(z)$ is of period $\pi$. So we need to show that $v$ is also of period $\pi \tau$.

From $(2.2)$,

$$
\frac{\theta_{1}\left(z+\frac{k \pi}{p}+\pi \tau \mid \tau\right)}{\theta_{1}(z+\pi \tau \mid \tau)}=e^{\frac{2 i k \pi}{p}} \frac{\theta_{1}\left(z+\frac{k \pi}{p} \mid \tau\right)}{\theta_{1}(z \mid \tau)} .
$$

Hence $v(z)$ is of period $\pi \tau$ if $2 \sum_{k \in R}(k / p)$ is even. To show this, we observe that since $p \geq 7, w=2$, and so from (2.10),

$$
2 \sum_{k \in R} \frac{k}{p}=\frac{p-1}{2}-h,
$$

and since $p \equiv 3 \bmod 4$ and $h$ is odd, (2.11) is even.

Remark 2. We begin with an observation:

$$
\begin{aligned}
\sum^{\prime}(-3 \mid n) \frac{n q^{n}}{1-q^{3 n}}= & \sum(-3 \mid n) \frac{n\left(q^{n}-q^{2 n}\right)}{1-q^{3 n}} \\
& =\sum(-3 \mid n) n \sum q^{(3 k-2) n}-q^{(3 k-1) n} \\
& =\sum(-3 \mid n) n \sum(-3 \mid m) q^{m n} \\
& =\sum \sum(-3 \mid n)(-3 \mid m) n q^{m n} \\
& =\sum(-3 \mid k) q^{k} \sum_{n \mid k} n \\
& =\sum(-3 \mid k) \sigma_{1}(k) q^{k}
\end{aligned}
$$

where $\sigma_{1}(n)$ is the sum of the divisors of $n$.

Differentiating $\varphi\left(z \mid \frac{D \tau}{2}\right)$ twice, we obtain

$$
\begin{aligned}
\varphi^{\prime \prime}\left(z \mid \frac{D \tau}{2}\right) & =i \frac{w}{4} \sum^{\times}(-D \mid k)\left(\frac{\theta_{1}^{\prime}}{\theta_{1}}\right)^{\prime}\left(z+\frac{k \pi \tau}{2} \mid \frac{D \tau}{2}\right) \\
& =-2 w \sum n Q_{D}\left(q^{n}\right) \sin 2 n z .
\end{aligned}
$$

For $D=3$, we have

$$
\begin{gathered}
\left(\frac{\theta_{1}^{\prime}}{\theta_{1}}\right)^{\prime}\left(z+\frac{\pi \tau}{2} \mid \frac{3 \tau}{2}\right)-\left(\frac{\theta_{1}^{\prime}}{\theta_{1}}\right)^{\prime}\left(z+\frac{2 \pi \tau}{2} \mid \frac{3 \tau}{2}\right) \\
=8 i \sum \frac{n\left(q^{n}-q^{2 n}\right)}{1-q^{3 n}} \sin 2 n z \\
=8 i \sum^{\prime} \frac{n q^{n}}{1-q^{3 n}} \sin 2 n z .
\end{gathered}
$$


Recall the additive formula:

$$
\left(\frac{\theta_{1}^{\prime}}{\theta_{1}}\right)^{\prime}(x)-\left(\frac{\theta_{1}^{\prime}}{\theta_{1}}\right)^{\prime}(y)=\frac{\theta_{1}^{\prime}(0) \theta_{1}(x-y) \theta_{1}(x+y)}{\theta_{1}^{2}(x) \theta_{1}^{2}(y)} .
$$

This formula is equivalent to the well-known identity [6, p. 451]:

$$
\wp(x)-\wp(y)=\frac{\sigma(x+y) \sigma(x-y)}{\sigma^{2}(x) \sigma^{2}(y)},
$$

where $\sigma(x)$ is the sigma function of Weierstrass.

Choose $z=\frac{\pi}{3}$ in (2.13). Then $\frac{\sin 2 n \pi}{3}=(-3 \mid n) \frac{\sqrt{3}}{2}$; and using Jacobi's triple product identity, we obtain an identity from $(2.12),(2.13)$ and (2.14) with $x=$ $\frac{\pi}{3}+\frac{\pi \tau}{2}$ and $y=\frac{\pi}{3}+\frac{2 \pi \tau}{2}$ :

$$
\sum(-3 \mid n) \sigma_{1}(n) q^{n}=\sum^{\prime}(-3 \mid n) \frac{n q^{n}}{1-q^{3 n}}=\frac{q\left(q^{9}, q^{9}\right)_{\infty}^{3}(q, q)_{\infty}^{3}}{\left(q^{3}, q^{3}\right)_{\infty}^{2}}
$$

where $(a, q)_{\infty}=\prod_{n=1}^{\infty}\left(1-a q^{n}\right)$.

\section{§3. An identity involving the Weierstrass $\wp$ FUnCtion And $\sigma_{1}(n)$}

The main goal of this section is to evaluate a certain sum of $\sigma_{1}(n)$ in terms of the values of the $\wp$ function at the rational points of the period parallelogram: $\left\{\frac{k \pi}{D}+\frac{n \pi \tau}{D}: 1 \leq k, n \leq D-1\right\}$. We will denote the $\wp$ function of periods $\pi$ and $\pi \tau$ by $\wp(z \mid \tau)$. The method used in deriving (2.12) can be generalized without changes to give the following identity:

$$
\sum k^{m} \chi(k) Q_{\chi, D}\left(q^{k}\right)=\sum \chi(k) \sigma_{m}(k) q^{k}
$$

for any character $\chi$ modulo $D$, where $\sigma_{m}(k)=\sum_{n \mid k} n^{m}$. This identity leads naturally to the consideration of the function

$$
\varphi_{\chi}\left(z \mid \frac{D \tau}{2}\right)=h z+\frac{w}{2} \sum \frac{1}{n} Q_{\chi, D}\left(q^{n}\right) \sin 2 n z .
$$

First, we need a simple lemma.

Lemma 3.1. If $\chi(-1)=-1$, then

$$
\sum^{\times} \bar{\chi}(k) \sin 2 n\left(z+\frac{k \pi}{D}\right)=-i g(\chi) \chi(n) \cos 2 n z .
$$

Proof.

$$
\begin{gathered}
\sum^{\times} \bar{\chi}(k) \sin 2 n\left(z+\frac{k \pi}{D}\right)=-\frac{i}{2} \sum^{\times} \bar{\chi}(k)\left(e^{2 i n z} e^{\frac{2 i k n \pi}{D}}-e^{-2 i n z} e^{\frac{-2 i k n \pi}{D}}\right) \\
=-\frac{i}{2} g(\chi) \chi(n)\left(e^{2 i n z}-e^{-2 i n z} \chi(-1)\right) \quad(\text { from (D)) } \\
=-i g(\chi) \chi(n) \cos 2 n z .
\end{gathered}
$$

Hence,

$$
\begin{aligned}
\sum^{\times} \bar{\chi}(k) \varphi_{\chi}^{\prime \prime}\left(z+\frac{k \pi}{D} \mid \frac{D \tau}{2}\right) & =-2 w \sum n Q_{\chi, D}\left(q^{n}\right) \sum^{\times} \bar{\chi}(k) \sin 2 n\left(z+\frac{k \pi}{D}\right) \\
& =2 i w g(\chi) \sum n \chi(n) Q_{\chi, D}\left(q^{n}\right) \cos 2 n z
\end{aligned}
$$

and from Lemma 2.1 we have

$$
\varphi_{\chi}^{\prime \prime}\left(z \mid \frac{D \tau}{2}\right)=i \frac{w}{4} \sum^{\times} \chi(n)\left(\frac{\theta_{1}^{\prime}}{\theta_{1}}\right)^{\prime}\left(z+\frac{n \pi \tau}{2} \mid \frac{D \tau}{2}\right) .
$$


Substituting (3.3) into (3.2) and setting $z=0$, we derive the following identity:

$$
\sum^{\times} \sum^{\times} \chi(n) \bar{\chi}(k)\left(\frac{\theta_{1}^{\prime}}{\theta_{1}}\right)^{\prime}\left(\frac{k \pi}{D}+\frac{n \pi \tau}{2}\right)=8 g(\chi) \sum \chi(n) \sigma_{1}(n) q^{n} .
$$

Recall the relation between $\theta(z \mid \tau)$ and $\wp(z \mid \tau)$ :

$$
\wp(z \mid \tau)=-\left(\frac{\theta_{1}^{\prime}}{\theta_{1}}\right)^{\prime}-\frac{1}{3} E_{2}
$$

where

$$
E_{2}=1-24 \sum \frac{n q^{2 n}}{1-q^{2 n}}
$$

Using (B) and (3.5), we derive the following theorem.

Theorem 2. Let $-D$ be the discriminant of an imaginary quadratic field, and let $\chi$ be a primitive character modulo $D$. Then

$$
\sum^{\times} \sum^{\times} \chi(n) \bar{\chi}(k) \wp\left(\frac{k \pi}{D}+\frac{n \pi \tau}{2} \mid \frac{D \tau}{2}\right)=8 g(\chi) \sum \chi(n) \sigma_{1}(n) q^{n} .
$$

We note that the first half of the identity (2.15) is a special case of Theorem 2 corresponding to $D=3$.

\section{§4. ElLiptic FUnCtion theORY ASSOCIATED With the IMAGinARY QUADRATIC FIELD}

In view of the crucial role played by the class number formula $(C)$, we can regard $\varphi^{\prime}\left(z \mid \frac{D \tau}{2}\right)$ and $\exp \left(-2 i \varphi\left(z \mid \frac{D \tau}{2}\right)\right)$ as elliptic functions associated with the imaginary quadratic field of discriminant $-D$. Furthermore, it should be pointed out that $\varphi^{\prime}\left(0 \mid \frac{D \tau}{2}\right)$ possesses an interesting arithmetic property connecting quadratic forms, the Kronecker symbol and the class number in a single Lambert series:

$$
\begin{aligned}
a_{D}:= & \varphi^{\prime}\left(0 \mid \frac{D \tau}{2}\right)=h+w \sum Q_{D}\left(q^{n}\right) \\
& =h+w \sum^{\infty}(-D \mid n) \frac{q^{n}}{1-q^{n}} \\
& =\sum_{Q} \sum_{m, n=-\infty}^{\infty} q^{Q(m, n)},
\end{aligned}
$$

where $Q$ runs through $h$ inequivalent quadratic forms of discriminant $-D$. See $[5$, p. 123, Theorem 4] for the proof of this fact.

Thus, for $D=3$ and 4 , we have

$$
\begin{aligned}
& a_{3}=\sum_{m, n=-\infty}^{\infty} q^{m^{2}+m n+n^{2}}=1+6 \sum(-3 \mid n) \frac{q^{n}}{1-q^{n}}, \\
& a_{4}=\sum_{m, n=-\infty}^{\infty} q^{m^{2}+n^{2}}=1+4 \sum(-4 \mid n) \frac{q^{n}}{1-q^{n}} .
\end{aligned}
$$

It is well known, from the general theory of elliptic functions, that any two elliptic functions $f$ and $g$ (of the same periods) are algebraically dependent; that is, there exists a polynomial $P(X, Y)$ such that $P(f, g)=0$. Therefore, from the Implicit Function Theorem, $g(z)=F(f(z))$ for some algebraic function $F$. 
Choose $f=f_{D}$ and $g=g_{D}$ as follows:

$$
\begin{aligned}
& f_{D}(z)=\sin ^{2} \varphi\left(z \mid \frac{D \tau}{2}\right)=\frac{1}{2}-\frac{1}{4}\left\{\exp \left(2 i \varphi\left(z \mid \frac{D \tau}{2}\right)\right)+\exp \left(-2 i \varphi\left(z \mid \frac{D \tau}{2}\right)\right)\right\} \\
& g_{D}(z)=\frac{1}{a_{D}} \varphi^{\prime}\left(z \mid \frac{D \tau}{2}\right) .
\end{aligned}
$$

Then $g_{D}(0)=1$ and from Theorem $1, f_{D}$ is elliptic for every $D$. We choose these two functions for study primarily because when $D=3,4$, they correspond, respectively, to the Ramanujan and classical cases; and when $D \geq 7$, they have the same poles and all the poles are of order one and thus might lead to a simpler polynomial equation.

We now consider the cases for $D=3$ and 4 . By comparing the poles of $f_{D}(=X)$ and $g_{D}(=Y)$, for $D=3$ and 4 , we deduce that they satisfy, respectively, the polynomials

$$
4-Y^{3}-3 Y^{2}=4 k_{3} X
$$

and

$$
Y^{2}=1-k_{4} X .
$$

See 4 for the details and exact values of $k_{3}$.

For the case $D=4$, clearly $a_{4}=\left(\theta_{3}(0 \mid \tau)\right)^{2}$ and it is well known that [6, p. 492] $k_{4}=k^{2}$ ( $k$ is called the modulus of the elliptic function). From (4.2), we see that $\varphi\left(z \mid \frac{4 \tau}{2}\right)$ is the unique solution of the differential equation:

$$
\frac{\varphi^{\prime}}{a_{4}}=\left(1-k_{4} \sin ^{2} \varphi\right)^{\frac{1}{2}} \text { and } \varphi(0)=0
$$

Then

$$
a_{4} z=\int_{0}^{\varphi}\left(1-k_{4} \sin ^{2} x\right)^{-\frac{1}{2}} d x=\int_{0}^{\varphi}{ }_{2} F_{1}\left(\frac{1}{2}, \frac{1}{2} ; \frac{1}{2} ; k_{4} \sin ^{2} x\right) d x .
$$

Here we use the hypergeometric series to write: $(1-z)^{-\frac{1}{2}}={ }_{2} F_{1}\left(\frac{1}{2}, \frac{1}{2} ; \frac{1}{2} ; z\right)$.

We have derived the following theorem.

Theorem A. Define

$$
a_{4} z=\int_{0}^{\varphi}{ }_{2} F_{1}\left(\frac{1}{2}, \frac{1}{2} ; \frac{1}{2} ; k_{4} \sin ^{2} x\right) d x .
$$

Then

$$
\varphi(z)=\varphi\left(z \mid \frac{4 \tau}{2}\right)=z+2 \sum \frac{1}{n} \frac{q^{n}}{1+q^{2 n}} \sin 2 n z .
$$

To see the connection of Theorem A with the classical Jacobi elliptic functions, we let $t=\sin x$ in (4.3). Then we obtain the well-known elliptic integral from which $\operatorname{sn}(z, k)$ is defined [6] p. 492]:

$$
z=\int_{0}^{s n(z, k)}\left(1-t^{2}\right)^{-\frac{1}{2}}\left(1-k^{2} t^{2}\right)^{-\frac{1}{2}} d t .
$$

Therefore, from this perspective, we can regard the classical Jacobi elliptic functions as the class of elliptic functions associated with the imaginary quadratic field $Q(\sqrt{-1})$. 
In the case of $D=3$, it is observed in [3] and [4] that

$$
Y(X)=\frac{1}{{ }_{2} F_{1}\left(\frac{1}{3}, \frac{2}{3} ; \frac{1}{2} ; X^{2}\right)}
$$

is the unique solution of (4.1) with $Y(0)=0$. Therefore, with $X=f_{3}$ and $Y=g_{3}$, we see that $\varphi\left(z \mid \frac{3 \tau}{2}\right)$ is the unique solution of the differential equation:

$$
a_{3}={ }_{2} F_{1}\left(\frac{1}{3}, \frac{2}{3} ; \frac{1}{2} ; k_{3} \sin ^{2} \varphi\right) \varphi^{\prime}, \quad \varphi(0)=1 .
$$

From this, we derive a remarkable statement of Ramanujan.

Theorem B. Define

$$
a_{3} z=\int_{0}^{\varphi}{ }_{2} F_{1}\left(\frac{1}{3}, \frac{2}{3} ; \frac{1}{2} ; k_{3} \sin ^{2} x\right) d x .
$$

Then

$$
\varphi\left(z \mid \frac{3 \tau}{2}\right)=z+3 \sum \frac{1}{n} \frac{q^{n}}{1+q^{n}+q^{2 n}} \sin 2 n z .
$$

Therefore, we can view this theorem of Ramanujan as a result of the elliptic function theory associated with the quadratic field $Q(\sqrt{-3})$.

We now apply the general theory of elliptic functions to $f_{D}$ and $g_{D}$. Then $\varphi\left(z \mid \frac{D \tau}{2}\right)$ satisfies a differential equation:

$$
a_{D}=F_{D}\left(\sin ^{2} \varphi\right) \varphi^{\prime}, \quad \varphi(0)=1
$$

for some algebraic function $F_{D}$. Hence, in theory, for every imaginary quadratic field, there exists an analogue of Theorems A and B:

Theorem 3. Define

$$
a_{D} z=\int_{0}^{\varphi} F_{D}\left(\sin ^{2} x\right) d x .
$$

Then $\varphi(z)=h z+\frac{w}{2} \sum \frac{1}{n} Q_{D}\left(q^{n}\right) \sin 2 n z$.

\section{REFERENCES}

1. A. I. Borevich and I. R. Shafarevich, Number Theory, Academic Press, New York, 1966. MR 33:4001

2. H. Cohn, Advanced Number Theory, Dover, New York, 1980. MR 82b:12001

3. B. C. Berndt, S. Bhargava and F. Garvan, Ramanujan's theories of elliptic functions to alternative bases, Trans. Amer. Math. Soc. 347(11) (1995), 4163-4244. MR 97h:33034

4. Li-Chien Shen, On an identity of Ramanujan based on the hypergeometric series ${ }_{2} F_{1}\left(\frac{1}{3}, \frac{2}{3} ; \frac{1}{2} ; x\right)$, J. of Number Theory 69(2) (1998), 125-134. MR 99d:11042

5. Li-Chien Shen, On a class of q-series related to quadratic forms, Bulletin of the Institute of Mathematics, Academia Sinica, 26(2) (1998), 111-126. MR 99h:11091

6. E. T. Whittaker and G. N. Watson, A Course of Modern Analysis, 4th edition, Cambridge University Press, London, 1958. MR 31:2375

Department of Mathematics, University of Florida, Gainesville, Florida 32611-2082

E-mail address: shen@math.ufl.edu 